
approches théoriques et méthodologiques

\title{
Organisation et architecture des connaissances dans un contexte de transmédia documentaire : les enjeux de la pervasivité
}

Architecture and organization of knowledge in the context of transmedia document: The challenges of pervasiveness

\section{Manuel Zacklad}

\section{CpenEdition}

Journals

Édition électronique

URL : http://journals.openedition.org/edc/4017

DOI : 10.4000/edc.4017

ISSN : 2101-0366

Éditeur

Université Lille-3

\section{Édition imprimée}

Date de publication : 1 décembre 2012

Pagination : 41-63

ISBN : 978-2-917562-08-6

ISSN : $1270-6841$

\section{Référence électronique}

Manuel Zacklad, « Organisation et architecture des connaissances dans un contexte de transmédia documentaire : les enjeux de la pervasivité ». Études de communication [En ligne], 39 | 2012, mis en ligne le 01 décembre 2014, consulté le 01 mai 2019. URL : http://journals.openedition.org/edc/4017 ; DOI : 10.4000/edc.4017

Ce document a été généré automatiquement le 1 mai 2019.

(c) Tous droits réservés 


\title{
Organisation et architecture des connaissances dans un contexte de transmédia documentaire : les enjeux de la pervasivité
}

\author{
Architecture and organization of knowledge in the context of transmedia \\ document: The challenges of pervasiveness
}

Manuel Zacklad

\section{Introduction}

1 L'Organisation des Connaissances (OC) occupe une place de plus en plus centrale dans la gestion de l'information numérique (Hjørland, 2008; Gnoli et Mazzocchi, 2010). Son rôle ne concerne plus seulement les usagers des bibliothèques ou des centres de documentation et il ne concerne plus seulement les fonds documentaires professionnels. Avec la numérisation généralisée de l'ensemble des média (photographie, musique, vidéo) comme avec la généralisation sans précédent de la production de documents pour l'action (DOPA) (Zacklad, 2004) qui peuvent circuler ou être stockés à des stades d'élaboration encore primitifs entre des postes de travail ou sur le web, la nécessité de disposer de techniques de documentarisation basées sur des Systèmes d'organisation des connaissances (SOC) (jeux de métadonnées, facilités de nommage, facilités de classement) permettant la mémorisation de l'information à court, moyen ou long terme dans toutes les applications informatiques, sur le poste de travail ou sur le réseau, pour les professionnels ou les particuliers, prend une nouvelle actualité.

2 Simultanément, la numérisation généralisée des supports et la fragmentation des contenus qu'elle rend possible ont considérablement complexifié la donne. La fragmentation des contenus permet de détacher un article de son support qu'il s'agisse d'un morceau de musique ou d'un article de journal, pour permettre des opérations de 
redocumentarisation éditoriale (Zacklad, 2008), les fragments documentaires réassemblés pouvant constituer de nouveaux documents autonomes. Ces processus de fragmentation et de recomposition accroissent les phénomènes de transmédialité documentaire et leur hétérogénéité dans le sens que nous définirons plus bas.

Dans cet article, nous défendrons l'idée selon laquelle ces nouveaux phénomènes impliquent une réorientation des recherches dans le domaine de l'OC qui tienne compte simultanément de la fluidité accélérée des contenus et de la complexité des architectures. Parmi ces recherches, certaines devront notamment enrichir le cadre conceptuel qui permet de rendre compte des relations entre les flux de DOPA de plus en plus multimédia et distribués et les modalités d'organisation des connaissances qui visent à préserver leur cohérence. Dans cette optique, nous présenterons un certain nombre de définitions et de concepts.

4 Après un retour sur les notions de contenu et de support, nous introduirons la notion d'environnement-support, matériel, applicatif, système et réseau d'un média. Nous préciserons ensuite les frontières existant entre les notions d'organisation des connaissances, de système d'organisation des connaissances, d'expre ssion sémiotique des connaissances et d'architecture des connaissances. Nous introduirons alors la notion de transmédia documentaire et ses déclinaisons, puis celle de pervasivité appliquée aux contenus et à l'organisation des connaissances qui les structure. Ces notions nous permettront d'interpréter certains résultats de recherche du projet ANR Miipa-Doc (Miipa-Doc n² 2008 CORD 014 03) qui vise précisément à accroitre la pervasivité de l'organisation des connaissances dans un contexte transmédia.

\section{Contenu et support des média : axiomes d'inséparabilité et d'incommensurabilité}

Comme le rappelle Bachimont (2009), dans les usages courants, si la notion de medium peut désigner " le support matériel d'une transmission ou d'une inscription ", le terme de média renvoie le plus souvent aux «moyens économiquement et industriellement organisés de diffusion, comme la télévision ou la presse ». Selon notre définition, basée sur la sémiotique des transactions coopératives (Zacklad, 2010a), le terme de média peut avoir deux acceptions : il renvoie soit à la notion d'artefact médiateur coréalisé par les personnes engagées dans une transaction coopérative, soit à la notion de dispositif médiatique constitué par le réseau des artefacts et des personnes rendant cette transaction possible dans une veine proche de celle des travaux en médiologie également présente dans l'expression "média de masse " (Debray, 2000). Nous utiliserons aussi le terme de médium pour désigner d'un point de vue technique la dimension espace d'inscription du support.

Dans le cadre de cet article, l'utilisation du terme de média correspond aux artefacts médiateurs à dominante sémiologique. Nous nous appuierons sur les théories de P. Descola (2005) pour opposer une vision en extériorité des artefacts médiateurs et des sujets à une vision en intériorité. Alors que le support médiatique correspond à une description en extériorité, le contenu relève d'une description symétrique mais en intériorité. Les média numériques sont des média à couches dissociées dont le médium a deux types de dimensions, certaines perceptibles, d'autres internes. Nous nous appuyons pour introduire cette distinction sur les définitions de Bachimont. Les médiums 
perceptibles sont ceux qui précèdent le numérique. Ils sont définis de la manière suivante :

Les médiums perceptibles ont leurs support d'enregistrement et support d'appropriation confondus : le support sur lequel on lit est celui que l'on range et stocke; de même, leurs formes d'enregistrement et d'appropriation sont confondues: ce que l'on lit est bien ce que l'on a inscrit sur le support [...]. Le contenu est ainsi inhérent au support [...] (Bachimont, 2009).

Bachimont oppose les médiums perceptibles aux médiums technologiques :

Les médiums technologiques ont un support d'enregistrement distinct et séparé du support de restitution. Ce que l'on conserve n'est pas ce que l'on consulte, car il est nécessaire de recourir à une médiation technique et mécanique pour accéder au contenu.

Ainsi, il ne suffit pas de conserver le support d'enregistrement pour accéder au contenu, il faut également conserver les conditions d'accès technique à l'information enregistrée pour la restituer dans une forme immédiatement consultable et interprétable (Bachimont, 2009).

8 Sur cette base, Bachimont oppose les supports d'enregistrement, par exemple un disque dur, et les supports de restitution, par exemple un écran. Nous nous inspirons de ces définitions en les transposant aux composants des média numériques. Ceux-ci sont des média à couches dissociées dans lesquels on peut distinguer des couches internes permettant le stockage des données et leur manipulation, et des couches perceptibles associées aux interfaces homme-machine. Les couches internes du médium incluent, par exemple, aussi bien le disque dur, la base de données et le logiciel d'interrogation. Les couches perceptibles du médium incluent aussi bien l'écran permettant l'affichage de l'information, les objets graphiques qui composent les métaphores de l'interface hommemachine et les programmes qui les animent en réponse aux évènements déclenchés par les gestes de l'utilisat eur.

Indépendamment du médium à couches dissociées, nous identifions deux autres dimensions du support, ce qui fait trois composantes: (1) le médium en tant qu'espace d'inscription (ou support au sens restreint) à l'intérieur duquel on distingue les couches perceptibles et les couches internes, (2) les inscriptions matérielles (ou contenu matériel) et (3) les procédés mécaniques permettant de faire circuler ce médium entre différents espaces. Par exemple, le support d'un message électronique contient (1) les médiums perceptibles correspondant à l'écran et aux interfaces homme-machine et les médiums internes correspondant au format de données et aux supports de stockage, (2) les inscriptions correspondant aux chaines de caractères véhiculant la signification dans le format de données et (3), l'ensemble des procédés permettant l'exécution des programmes dans un environnement local mais également distribué permettant notamment la circulation du message dans un écosystème technologique plus vaste (Figure 1).

10 Cette description du support nous permet par contraste de définir la notion de contenu qui peut se décomposer elle aussi en trois composantes: (1) la signification qui, dans le cas des artefacts sémiologiques, correspond directement aux inscriptions, (2) les modalités expressives associées au médium relevant d'un genre spécifique auquel sont associées des conventions sémantiques et sémiotiques, (3) les effets potentiels sur les réalisateurs et les bénéficiaires dans une perspective de pragmatique linguistique. 
Figure 1 : Décomposition du média dans son acception d'artefact médiateur

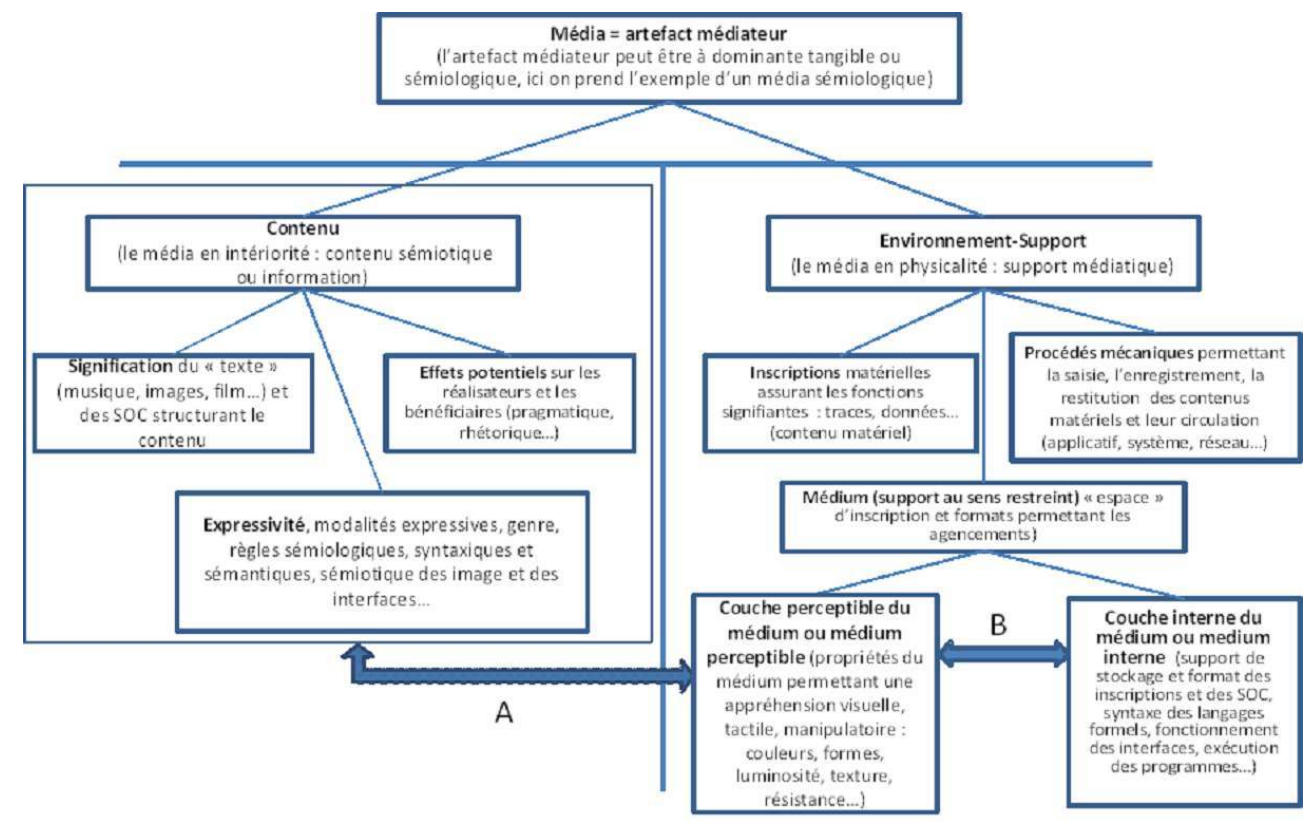

11 Bien entendu, aucun contenu ne peut être accessible sans un support et celui-ci conditionne toujours les modes d'appréhension de manière singulière. La distinction « contenu-support » résulte toujours d'une abstraction, le format de compression, comme la taille de l'écran pour ne prendre que deux exemples, conditionnent de manière drastique les formes d'appréhension des contenus. Ceci correspond à l'axiome d'inséparabilité matérielle du contenu vis-à-vis du support :

12 La distinction contenu-support est une distinction fonctionnelle qui n'a pas de portée ontologique. Un contenu n'a pas d'existence indépendamment du support qui le véhicule. Le contenu véhiculé par un média n'est assimilable à un autre contenu qu'en faisant abstraction de leurs différences matérielles (même infimes). Un contenu peut être considéré comme «quasi-identique » quand il est reproduit industriellement sur les différents exemplaires d'un même

type de support lui-même produit industriellement selon des normes de qualité poussées. Les dispositifs de restitution des inscriptions matérielles et le contexte dans lequel celleci est effectuée, toujours variable, influencent également la réception d'un contenu. Un jugement d'identité entre deux contenus est toujours le résultat d'une activité cognitive d'interprétation.

14 Dans les média numériques à couches dissociées, la question du couplage entre les couches se pose avec acuité. On peut identifier différentes relations de couplage entre le contenu et le support (flèche A, Figure 1) et entre les médiums perceptibles et internes du support (flèche B, Figure 1) et on distinguera un couplage faible et un couplage fort. Le couplage contenu-support est essentiellement réalisé pour l'utilisateur à travers le média perceptible. Dans le contexte des interfaces à manipulation directe qui sont devenues la norme, il correspond aux choix de bonnes métaphores graphiques facilitant l'apprentissage des commandes et l'exploration des données (les inscriptions matérielles associées à la signification). On parlera d'un couplage fort quand il existe un fort isomorphisme entre les pratiques d'écriture, de lecture, de manipulation d'objets au sens large des utilisateurs dans un contexte applicatif donné et les métaphores graphiques qui 
représentent les commandes et l'organisation des données. Ces pratiques elles-mêmes sont en constante évolution et tiennent compte des habitudes $d$ es usagers dans la manipulation des versions antérieures des logiciels. Ces enjeux renvoient à des considérations de qualité ergonomique et d'usage.

La deuxième relation de couplage se situe entre les couches perceptibles et internes du médium (B). Les informations qui apparaissent dans l'interface homme-machine peuvent être plus ou moins fortement couplées à la structure de données interne. On peut donner l'exemple du regroupement des icônes représentant des ressources (souvent des fichiers) sur le "bureau» des postes de travail : les associations topologiques entre icônes ont souvent une signification classificatoire pour les utilisateurs mais celles-ci n'étant pas capturées par le système, ce dernier peut proposer des réorganisations automatiques de l'affichage qui ne tiennent pas compte du sens conféré par les usagers.

16 A la multiplication des couches correspond également une multiplication des modalités d'organisation des connaissances associées: l'OC peut être ancrée dans les couches internes du médium et faire l'objet d'une codification explicite dans les systèmes de métadonnées tout en étant invisible pour les utilisateurs; l'OC peut posséder les mêmes propriétés mais être associée à des listes perceptibles renvoyant directement aux propriétés internes; l'OC peut être directement projetée dans des métaphores imagées utilisées dans l'interface homme-machine.

Plus les relations sémiotiques entre les contenus véhiculées par l'interface hommemachine, par exemple une relation de regroupement, sont explicitées sémantiquement dans les couches internes, plus le couplage entre les différentes couches du médium sera considéré comme fort. Mais, quelle que soit la force de ce couplage, l'organisation des connaissances portée par les métaphores graphiques associées aux interfaces hommemachine excèdera toujours les possibilités de la formalisation sémantique interne. Il s'agit d'un deuxième axiome indispensable à la compréhension des relations contenu-support qui s'applique ici aux média à couches dissociées, l'axiome d'incommensurabilité de l'organisa tion des connaissances sémiotiques :

18 Une organisation des connaissances sémiotique portée par une métaphore graphique est incommensurable à toutes formalisations sémantiques sous-jacentes. Cette incommensurabilité est liée au sens conféré par les utilisateurs à la métaphore, sens qui s'ancre dans des pratiques culturelles diverses et évolutives.

\section{Environnement-support matériel, applicatif, système et réseau}

19 Les média numériques mobilisent quatre types d'environnements-supports imbriqués pour rendre possible l'accès aux inscriptions et donc aux contenus. Les enjeux de compatibilité entre les formats de données et les programmes utilisés par ces différents environnements-supports sont au cœur de nombreuses rivalités industrielles et commerciales.

20 Le premier environnement est l'environnement-support matériel qui fournit les ressources en mémoire et en traitement à tous les média numériques. Avec le développement sans précédent des terminaux mobiles connectés à Internet, dont la société Apple a lancé le grand mouvement de généralisation, l'accès en mobilité qui était jusqu'alors essentiellement limité à la musique et aux jeux vidéo s'est généralisé à tous les 
média, y compris en mode "création». De nombreux facteurs interviennent pour caractériser l'environnement-support matériel : la taille de l'écran, le dispositif de saisie (clavier-souris ou tactile...), la puissance du processeur, la vitesse de la connexion internet, etc. Les nouveaux « terminaux » popularisés avec la console Kinect de Microsoft utilisent une caméra comme dispositif de saisie et on peut imaginer prochainement la généralisation de terminaux remplaçant l'écran par d'autres dispositifs de visualisation intégrés au mobilier.

21 Le deuxième environnement-support est l'environnement-support applicatif qui permet de restituer les données dans une forme perceptible pour les utilisateurs. Chaque application correspond au traitement d'un problème spécifique qui se traduit par des modalités particulières de lecture et d'écriture. Le même support d'inscription, support au sens restreint, correspondant à un fichier ou à un champ d'une base de données, peut être pris en charge par différents environnements applicatifs selon les besoins. Souvent, certaines applications ne servent q ue de "liseuses » pour des fichiers de différents formats en permettant de rajouter des annotations sans qu'il soit possible d'éditer le contenu en profondeur.

Le troisième est l'environnement-support système qui correspond au système d'exploitation et qui est directement lié au terminal dans lequel tournent les applications. L'environnement-système est également un média à couches dissociées qui contient des couches perceptibles basées sur des métaphores graphiques et des couches internes. Parmi les composants du système d'exploitation figurent le système de gestion de fichiers qui gère les arborescences et prend notamment en charge l'inscription d'un nombre important de métadonnées et l'affichage de différentes vues permettant de gérer des collections de fichiers importantes. Le système de gestion collabore étroitement avec les environnements applicatifs en permettant, par exemple, une prévisualisation des fichiers pendant la navigation dans les arborescences.

23 Enfin, le quatrième est l'environnement-support réseau. Aujourd'hui cette infrastructure est largement basée sur le web, y compris dans des contextes intra-entreprise. L'environnement réseau permet des échanges de données en temps quasi réel entre les applications en mobilisant différents protocoles dont http est le principal. L'environnement réseau possède lui-même une application préférée qui tend à concentrer l'accès à un nombre très important de données: le navigateur. Pour des raisons de sécurité, celui-ci est d'ailleurs relativement dissocié du gestionnaire de fichiers, ce qui ne facilite pas les pratiques des utilisateurs. Les navigateurs sont également des applications à couches dissociées exploitant différentes métaphores graphiques et ils accueillent en leur sein de nombreux lecteurs traitant la plupart des formats de fichiers disponibles.

Les normalisateurs du web promeuvent une architecture basée sur la généralisation de la notion de ressource, un contenu matériel de taille variable (inscriptions), associé à une adresse web (une URI) et des métadonnées. Ils sont également attachés à l'explicitation des relations sémantiques entre les ressources sans toujours bien prendre en considération la diversité des couches dans lesquelles s'exprime l'OC ni l'axiome d'incommensurabilit_ 8e de l'organisation des connaissances sémiotique qui restreint de manière principielle les possibilités de codification logico-mathématique des connaissances. 


\section{Organisation et architecture des connaissances}

25

Alors que certains auteurs défendent la disparition de l'idée même de document sous la poussée des technologies du web et de la fragmentation des supports, nous prenons le parti inverse en considérant que cette notion, comme celle de dossier et de collection, permet au contraire de remettre de l'intelligibilité dans les fragments médiatiques qui circulent de manière de plus en plus rapide et automatisée. Selon notre définition (Zacklad, 2004), le document résulte d'un processus actif de documentarisation visant à permettre la ré-exploitation d'un support et des contenus qu'il véhicule. Le document est donc directement lié à un processus de mémorisation individuel ou collectif (de témoignage, de preuve, de soutien mnésique) dont le statut lui-même ne peut se comprendre que dans le contexte d'un projet transactionnel singulier (Briet, 1951; Meyriat, 1981 ; Zacklad, 2004 ; Pedauque, 2006).

26 Par exemple, si un fragment est extrait d'un document numérique, cette extraction qui n'est plus associée à l'organisation des connaissances d'ensemble qui lui donnait sens ne constitue plus un document. Associée à d'autres fragments issus d'autres supports documentaires, elle ne refera à nouveau sens, c'est-à-dire document, qu'à l'issue d'un autre processus de documentarisation, par exemple de nature éditoriale, qui confèrera aux différents fragments un sens nouveau dans le cadre d'un nouveau projet transactionnel associé à une nouvelle organisation des connaissances.

Mais tous les artefacts médiateurs mobilisés dans les transactions ne sont pas des documents, certains parce qu'ils ne sont pas bas_ 8es sur un support d'écriture ou d'enregistrement permettant cette forme d'appropriation particulière qu'est la documentarisation (des gestes ou des paroles), d'autres parce qu'ils n'ont pas pour finalité d'assister un processus informationnel ou mnésique ou qu'ils visent à l'assister mais en utilisant d'autres procédés. Par exemple, dans notre article de 2004, parmi les stratégies de coordination des "transactions communicationnelles distribuées", nous évoquions d'autres procédés tels que la normalisation de la situation transactionnelle, la formalisation de l'expression, la ritualisation mnémotechnique ou l'abstraction permettant de contribuer à la mémoire d'une transaction sans recourir au document.

Si la notion d'OC est au cœur des processus de documentarisation, nous donnons également à celle-ci une extension qui excède les usages documentaires. Il existe des modalités d'OC associées à toutes les formes d'expression : parole, dessin, danse, musique, manipulation gestuelle... Si les connaissances correspondent pour nous aux artefacts médiateurs internalisés et externalisés qui permettent de réaliser une "performance ", l'OC correspond aux procédés permettant la structuration de ces connaissances de manière à faciliter leur mémorisation, leur évocation et leur exploitation (attention, perception, raisonnement, mémoire).

29 Pour l'essentiel, une organisation des connaissances peut être assimilée à un plan du contenu, plan remplissant les deux fonctions classiques de schématisation et d'aide à l'anticipation temporelle correspondant aux dimensions déclaratives et procédurales (Hoc, 1987). Selon la première dimension, toute organisation des connaissances contient une dimension classificatoire en opérant des catégorisations même partiellement implicites. Selon la deuxième dimension, toute organisation des connaissances relève d'une forme de programmation de l'action renvoyant notamment à la nécessité pour 1 es

Études de communication, 39 | 2012 
artefacts externes d'offrir des affordances guidant la manipulation des artefacts et permettant de découvrir leurs fonctions.

Si l'OC renvoie à la dimension du contenu, de l'information, l'architecture des connaissances renvoie elle à la dimension du support, de la physicalité, de la matérialité. Par exemple, si la conception d'une exposition obéit à un principe d'OC consistant à classer les œuvres selon une logique chronologique, l'architecture des salles d'exposition planifiera le parcours des visiteurs de manière à les conduire d'abord vers les œuvres chronologiquement antérieures. Selon cette perspective, l'architecture du bâtiment correspond à l'architecture des connaissances associées au projet d'exposition. L'OC chronologique va s'instancier dans différentes architectures de connaissances matérielles selon le $s$ supports: table des matières du catalogue d'exposition classifiant la reproduction des œuvres et programmant le parcours de lecture ou agencement des salles et répartition des œuvres en leur sein permettant de les classifier et de programmer le parcours de visite.

L'organisation des connaissances donne lieu généralement à l'élaboration d'un «langage» qui permettra, par exemple, d'expliciter un schéma de classification ou d'indexer des ressources. Dans notre exemple, la perspective chronologique sera associée à des termes contrôlés décrivant la périodisation temporelle. Les langages qui permettent de rendre compte d'une organisation des connaissances ont reçu le nom de «système d'organisation des connaissances" (SOC) (Hodge, 2000). Ils sont utilisés pour la classification ou l'indexation dans la documentarisation interne et externe et incluent également pour nous des systèmes d'index construits automatiquement comme le sont ceux des moteurs de recherche (Zacklad, 2010b). C'est uniquement quand ils sont basés sur un langage que les SOC peuvent être mobilisés dans des architectures de connaissances numériques qui peuvent exploiter des formes d'association entre les termes pour les automatiser et faciliter la navigation. Dans le cas contraire, l'OC utilise des principes d'expression non langagiers et donne lieu à une expression sémiotique des connaissances, par exemple une représentation imagée, qui pourra également être inscrite sur un support, comme c'est le cas pour les métapho res graphiques utilisées dans les couches perceptibles des

Figure 2 : Organisation, système, expression, architecture des connaissances médias.

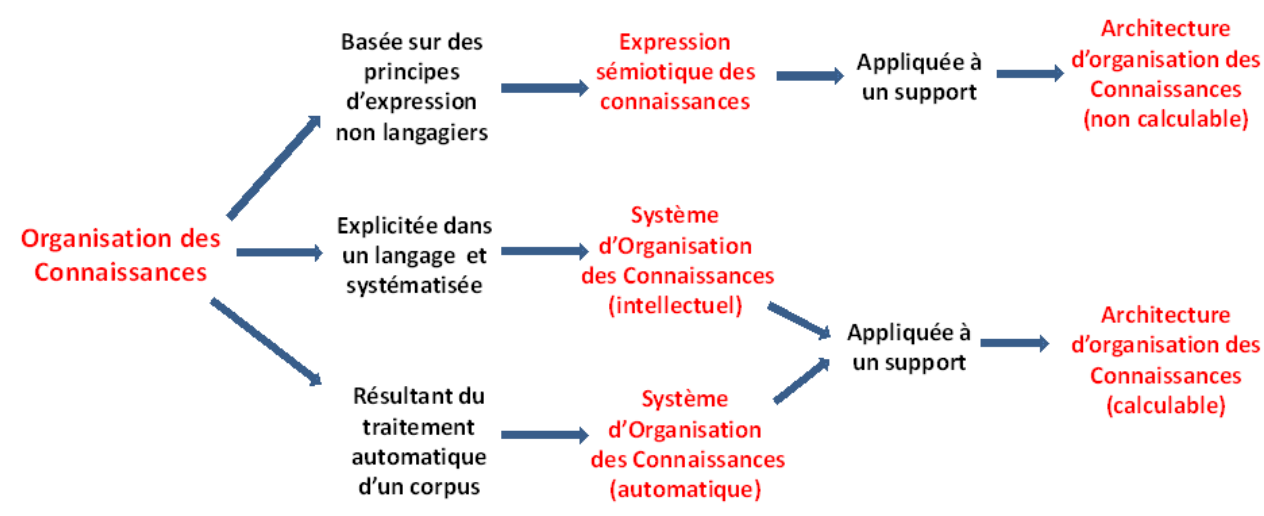




\section{Transmédia et transmédialité}

Bien que la problématique du transmédia soit issue de l'univers des loisirs numériques, elle est pourtant d'une actualité brûlante dans l'ensemble des contextes d'usage des média numériques en englobant à la fois les enjeux de multimodalité, contenus associant de manière complémentaire texte, audio, vidéo, etc., de pervasivité, circulation d'un contenu sur une multiplicité de terminaux et d'environnements applicatifs diffus, et enfin de convergence, concentration au sein du même terminal de plusieurs types de média jusqu'alors pris en charge par des terminaux différents. Selon Jenkins, un des premiers promoteurs de l'expression,

transmedia storytelling represents a process where integral elements of a fiction get dispersed systematically across multiple delivery channels for the purpose of creating a unified and coordinated entertainment experience. Ideally, each medium makes its own unique contribution to the unfolding of the story (2011).

Un des aspects les plus intéressants de l'approche transmédia est de considérer le rôle fonctionnel spécifique de chaque support dans un processus narratif cohérent qui ouvre la voie à la problématique d'une rhétorique des supports. Si l'on quitte les aspects purement fictionnels pour s'intéresser à des processus d'activité au sens large, notamment au sein des organisations, on entrevoit toute la portée de cette approche. Les processus peuvent être des processus de conception, actualisation progressive d'une représentation schématique, ou des processus de construction assemblant les composants nécessaires à l'atteinte d'un état ou d'un jugement final sur la base d'un plan déjà élaboré. Notons également qu'en référence à l'industrie des loisirs, dont cette notion est issue, Jenkins parle à la fois de mÄ edium et de canal de distribution.

Cette notion de canal de distribution renvoie chez nous à la notion de dispositif médiatique. Alors que celle-ci prend en compte différentes composantes du contexte transactionnel en mettant en évidence le réseau complexe d'artefacts médiateurs et d'acteurs contribuant à la performance, la notion de média en tant qu'artefact médiateur, se focalise sur la coproduction en situation du média en faisant en partie abstraction du contexte élargi. Nous distinguerons donc une acception du transmédia classique, transmédia "canal de distribution", d'une acception basée sur la notion d'artefact médiateur. Quand les artefacts médiateurs sont uniquement de nature documentaire, nous parlerons de transmédia documentaire et nous parlerons de transmédia hybride s'ils incluent d'autres artefacts médiateurs comme des conversations ou l'organisation d'évènements (Figure 3). 


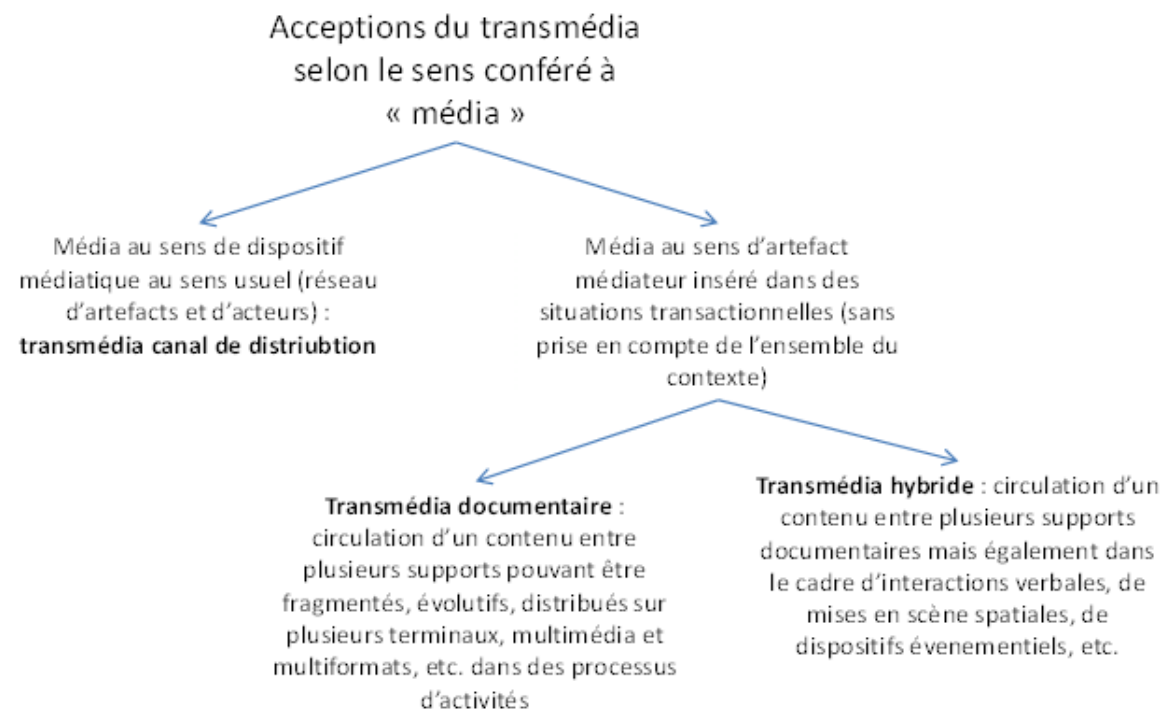

Il est de coutume de distinguer le plurimédia (ou cross-média) du transmédia. Alors que le premier reproduit un contenu identique sur plusieurs supports, le second mobilise plusieurs supports de manière complémentaire, chaque support « contribuant de manière unique au déroulement de l'histoire", pour reprendre les termes de Jenkins cité plus haut. Mais la transposition d'un support à l'autre ne pouvant pas ne pas avoir de conséquence sur le contenu lui-même, cette distinction est bien sûr relative (la transposition d'un livre dans un film modifie nécessairement la narration et donc le contenu de l'histoire).

Pour élaborer une typologie des types de transmédialité, on s'appuiera sur l'opposition faite par Jenkins entre deux pôles, celui de l'adaptation d'une narration, qui transpose une histoire d'un média à l'autre et celui de l'extension de la narration qui se prolonge sur différents média. Il y a un continuum entre ces deux pôles: alors que l'adaptation consiste en la réitération du même programme transactionnel dans des contextes différents, l'extension correspond à une prolongation d'un programme transactionnel dans lesquels la performance mobilise successivement différents artefacts médiateurs. Cette typologie peut s'appliquer aux artefacts médiateurs comme aux dispositifs médiatiques. Nous la mobilisons dans le cadre de l'analyse des transmédias documentaires (Tableau 1).

Tableau 1 : Différents types de transmédias documentaires

\begin{tabular}{|l|l|l|}
\hline $\begin{array}{l}\text { Contenu } \\
\text { Support }\end{array}$ & $\begin{array}{l}\text { Adaptation } \\
(\text { même contenu })\end{array}$ & $\begin{array}{l}\text { Extension } \\
\text { (contenus différents) }\end{array}$ \\
\hline
\end{tabular}




\begin{tabular}{|c|c|c|}
\hline $\begin{array}{l}\text { Même type } \\
\text { de support } \\
\text { de l'artefact } \\
\text { médiateur (et/ou } \\
\text { du dispositif } \\
\text { médiatique) }\end{array}$ & $\begin{array}{l}\text { Adaptation transmédia } \\
\text { Différentes versions d'un document } \\
\text { qui vise la même finalité (adaptation } \\
\text { transmédiatique d'un même contenu) }\end{array}$ & $\begin{array}{l}\text { Processus transmédia } \\
\text { Différents documents } \\
\text { complémentaires dans le même } \\
\text { environnement } \\
\text { système, réseau...) rassemblés dans } \\
\text { le cadre d'un dossier (déroulement } \\
\text { transmédiatique d'un processus) }\end{array}$ \\
\hline $\begin{array}{l}\text { Différents types } \\
\text { de support } \\
\text { des artefacts } \\
\text { médiateurs } \\
\text { (et/ou du } \\
\text { dispositif } \\
\text { médiatique) }\end{array}$ & $\begin{array}{l}\text { Transposition } \\
\text { transmédia } \\
\text { Des documents de format différent et } \\
\text { dans des environnements différents } \\
\text { (applicatif, système, réseau...) } \\
\text { contribuant à la même finalité } \\
\text { (transposition transmédiatique d'un } \\
\text { même contenu) : mails, fichiers, scan, } \\
\text { vidéo, SMS... }\end{array}$ & $\begin{array}{l}\text { Processus transmédia hétérogène } \\
\text { Différents documents } \\
\text { complémentaires de format } \\
\text { différent et dans des } \\
\text { environnements } \\
\text { (applicatif, système, réseau...) } \\
\text { contribuant à la même finalité }\end{array}$ \\
\hline
\end{tabular}

Dans le transmédia documentaire, les enjeux concernent aussi bien les différentes couches et environnements-supports du même média numérique (les médiums) que le développement d'un contenu à travers des média distincts. Ainsi, on parlera d'adaptation transmédia d'un contenu entres les différentes versions d'un document (Tableau 1) et de transposition transmédia lorsque que le même type de contenu est représenté dans des genres distincts (texte, photo, vidéo) ou à l'intérieur du même média numérique lors de la transposition partielle du contenu associé à une couche perceptible basée sur une métaphore graphique à une couche interne basée sur un format codé (par exemple avec un langage de balises, XML, HTML, etc.).

Quand différents documents numériques contribuent au développement d'un dossier en apportant des pièces complémentaires correspondant à des contenus spécifiques mais interreliés, la transmédialité relève du processus transmédia (Tableau 1). Enfin, quand ces différents documents sont également associés à différents environnements applicatifs (différents formats de fichiers), à différents environnements systèmes (fichiers répartis sur différents terminaux), ou à différents environnements réseaux (fichiers répartis sur différents serveurs non nécessairement reliés), la transmédialité correspond à un processus transmédia hétérogène (Tableau 1).

\section{Organisation des connaissances pervasive en environnement transmédia}

Avec l'augmentation du recours aux documents pour l'action (DOPA) qui tendent à associer des formats de plus en plus divers et une diversité de terminaux et de systèmes, les différentes configurations de transmédialité se généralisent au sein des organisations. Dans ce contexte, les enjeux de pervasivité de l'oC deviennent critiques. Classiquement, la pervasivité est associée à l'informatique ubiquitaire, c'est-à-dire à une informatique qui n'est plus limitée aux frontières du poste de travail mais qui est de plus en plus incorporée aux objets de la vie quotidienne (on parle d'aussi d'intelligence ambiante). 

sa capacité à circuler à travers différents média et relève donc d'enjeux liés à la transmédialité au sens où nous l'avons évoqué plus haut. La pervasivité d'une organisation des connaissances correspond à la manière dont cette organisation des connaissances pourra être projetée dans différents média dans une optique d'adaptation, projection de contenus similaires sur des supports identiques ou distincts, ou d'extension, projection de contenus évolutifs dans le cadre d'une activité sur des supports identiques ou distincts.

Cette pervasivité pourra être abordée en intériorité, du point de vue du contenu, ou en extériorité, du point de vue du support. Dans le premier cas, elle dépend de la capacité de l'OC à s'adapter aux différents contextes dans lesquels le média est utilisé, comme de sa capacité à évoluer au fur et à mesure que le contenu évolue, que cette évolution se situe à l'intérieur du même média ou traverse plusieurs média liés par une relation narrative, argumentative ou d'actualisation (dans un processus de conception). Cette évolutivité de l'organisation des connaissances dépendra des caractéristiques des SOC: richesse des relations d'association entre les termes (de nature typée et référentielle, heuristique ou statistique), capacité à représenter des points de vue multiples voire contradictoires, évolutivité des termes.

La pervasivité d'une organisation des connaissances du point de vue du support dépend de la capacité de l'organisation des connaissances associée à un contenu à s'appliquer aux différents média qui véhiculent ce contenu ou aux différentes couches d'un média à couches dissociées. Ceci renvoie directement aux enjeux d'architecture des connaissances qui se posent de manière différentes dans un environnement applicatif, système ou réseau.

\section{Prise en charge de la pervasivité de l'organisation des connaissances du point de vue du contenu et du support}

Pour illustrer les enjeux de la pervasivité de l'oc dans un contexte de transmédia documentaire, nous prendrons quelques exemples issus de l'application de documentarisation classificatoire et de recherche d'information multidimensionnelle Hypertagging et du Composant d'Infrastructure de Classement et d'Indexation (CICI) qui lui est associée, développés dans le cadre du projet ANR Miipa-DOC (Zacklad et al., 2011). Du point de vue du contenu, nous avons vu que les enjeux de la pervasivité de l'OC dans les processus transmédia impliquent de recourir à des SOC suffisamment riches pour rendre compte des différentes situations d'activité et suffisamment flexibles pour intégrer leurs évolutions.

Dans le cadre du projet Miipa-Doc, nous avons fait le choix de recourir à la classification à facettes en combinant facettes individuelles et collectives et en rendant possible à la fois la création de nouvelles facettes et une évolution de leurs valeurs (Zacklad et al., 2011; Desfriches-Doria, 2012). Les facettes sont elles-mêmes regroupées dans des classeurs correspondant à des contextes d'activité et le même média peut être appréhendé dans ces différents contextes. Nous nous appuyons également sur des icônes pour renforcer l'affordance perceptive des systèmes de facettes. 

sans parvenir toutefois à proposer des solutions qui permettraient de gérer l'ensemble des contextes de transmédialité documentaire tant la situation est complexe. Nous avons développé deux types de fonctionnalités, les unes visant à propager la même organisation des connaissances dans la gestion du classement, de l'indexation et du nommage, les autres visant à prendre en compte la mobilité des documents numériques entre plusieurs environnements système et réseau.

Un des problèmes d'usage essentiel auquel sont confrontés les utilisateurs des environnements documentaires numériques est lié à l'hétérogénéité des classifications documentaires qui leur sont proposées et à leur caractère redondant et parfois conflictuel. Toutes les formes de transmédialité documentaire sont mises à mal par ce phénomène depuis la simple adaptation transmédia (voir les travaux de Chabin, 2010, sur les versions) jusqu'aux processus transmédia complexes qui entrainent des familles de documents dans des circuits intriqués et parfois difficilement prévisibles. Les solutions proposées par les grands éditeurs de GED (Gestion Electronique de Documents) sont basées sur le présupposé selon lequel tous les utilisateurs recourent de manière systématique et disciplinée à la base de donnée s pour indexer les documents.

Or, ces scénarios sont largement irréalistes. Comme nous le développons dans notre article de 2011, une grande partie des activités de classification documentaire dans la gestion opérationnelle des documents pour l'action est effectuée dans les répertoires de classement individuels ou collectifs situés dans les environnements système et réseau qui ne sont généralement pas directement connectés à l'environnement applicatif de la GED. En d'autres termes, une part importante de la classification est réalisée dans le cadre des opérations de classement, directement dans l'environnement système c'est-à-dire dans les arborescences de dossiers. L'application Hypertagging, développée dans le cadre du projet ANR Miipa-Doc, fournit une interface de documentarisation classificatoire unique qui permet une semi-automatisation des opérations de nommage, de classement et d'indexation en garantissant leur cohérence. Elle se substitue à l'exploration des hiérarchies de répertoires comme aux opérations d'indexation dans les systèmes de GED et les CMS qui impliquent des opérations de saisie réalisées à travers un navigateur (Figure 4). 
Figure 4 : Interface de classement de l'application Hypertagging

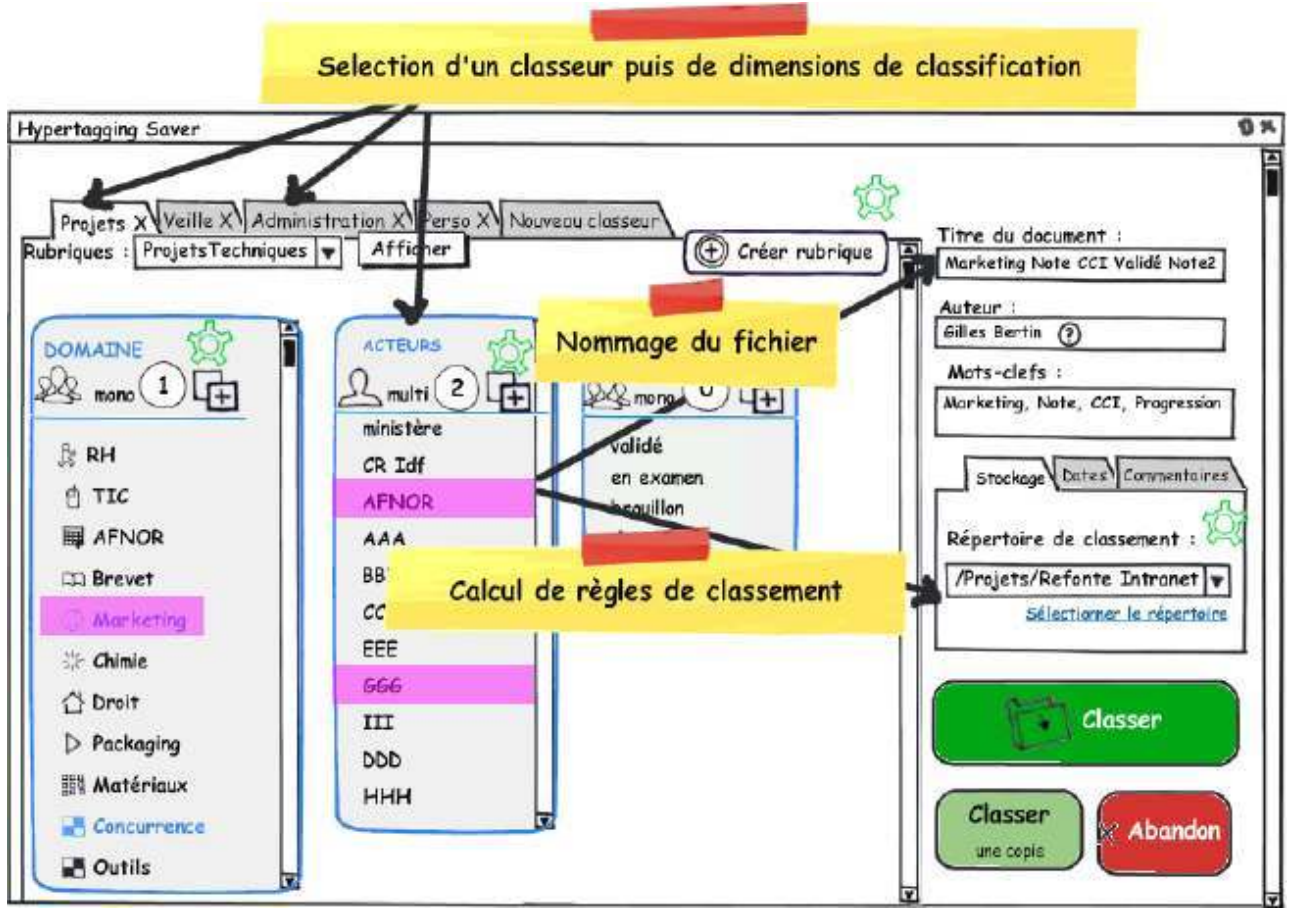

La deuxième difficulté, que l'application Hypertagging ne peut pas traiter par elle-même, est liée à la mobilité des fichiers. Les utilisateurs déplacent manuellement les fichiers à l'intérieur de leur environnement système mais également entre plusieurs environnements systèmes, à l'aide de la messagerie électronique ou d'une clef USB. Ils le font, par exemple, dans le cadre de processus transmédia, pour regrouper dans le même environnement système plusieurs documents liés afin d'y accéder de manière synchronisée. Une des solutions que nous avons explorées dans le CICI (Composant d'Infrastructure de Classement et d'Indexation) consiste à écrire les métadonnées utilisées pour l'indexation classificatoire à l'intérieur des fichiers puis à utiliser l'index du moteur de recherche pour les retrouver. En effet, 1 es moteurs de recherche traitent de manière spécifique les métadonnées et les gèrent de manière efficace et non-ambigüe.

Quand un fichier est déplacé dans un nouvel environnement système accessible par un moteur de recherche, son index peut perdre d'abord la trace du fichier, mais il sera rapidement en mesure d'être mis à jour grâce aux processus d'exploration (crawl) intégrés dans le moteur. Lors de la recherche d'information, l'application HyperTagging adresse directement ses requêtes à l'index du moteur de recherche en utilisant les API disponibles. Grâce à ce mécanisme, la pervasivité de l'organisation des connaissances est assurée à l'intérieur de plusieurs environnements systèmes. Mais ce mécanisme a également des limites. En effet, l'écriture des métadonnées d'indexation à l'intérieur des fichi ers pose plusieurs problèmes : des problèmes d'intégrité du fichier et des problèmes liés à la diversité des formats et des applications, certains formats ne permettant pas, par exemple, l'écriture des métadonnées dans des zones réservées. 


\section{Conclusion : pour des recherches en $\mathrm{OC}$ intégrant les contraintes du transmédia}

49 La soi-disant dématérialisation qui devait entrainer l'indépendance du contenu et du support débouche en fait sur une complexification considérable des phénomènes transmédiatiques qui accroissent les difficultés liées à l'organisation des connaissances du point de vue des contenus et de l'architecture des supports. Quand les supports étaient perceptibles, au sens de Bachimont (2009), les évolutions transmédia adaptatives des versions d'un document se traduisaient par l'ajout de commentaires multiples dans la marge de la page d'un exemplaire unique et les processus transmédia étaient gérée dans des dossiers physiques à l'aide de procédés d'attachement divers: classeurs, agrafes, trombones, élastiques...

50 La poussée vers la pervasivité des contenus a fait exploser les frontières du poste de travail qui est devenu pour partie une extension du web et a multiplié le nombre de terminaux et de systèmes pour faciliter l'accès en mobilité aux contenus multimédia. Mais chaque terminal a été doté de son propre environnement système souvent incompatible avec les environnements applicatifs tiers. Du côté du web, dont l'importance s'est encore accrue avec le stockage en mode «cloud» (infonuagique), la guerre des standards entre les navigateurs n'a pas cessé (Le Crosnier et Lecarpentier, 2010) et les environnements applicatifs de gestion de contenu et d'édition documentaire disponibles sur le nuage se sont multipliés. Cette fragmentation, doublée d'une multiplication des environnements applicatifs, accroit notablement l'hétérogénéité transmédia.

51 Sur cette architecture documentaire, ou architecture de l'information, considérablement complexifiée, les nouvelles formes d'organisation des entreprises et les pratiques collaboratives poussant à la multiplication de DOPA de toutes natures sont devenues la norme. Ces documents s'inscrivent dans des logiques de travail par projet et d'innovation en continu qui poussent eux-mêmes à une évolution permanente des contenus, de leurs thématiques et des logiques organisationnelles qui leur sont associées. Face à cette double évolution, la nécessité de disposer de SOC qui soient à la fois suffisamment souples pour refléter la fluidité des contenus et assez pervasifs pour s'adapter à la complexité des architectures est une nécessité pour les individus comme pour les organisations.

Or, la recherche en organisation des connaissances nous semble encore trop marquée par ses origines liées aux bibliothèques de recherche, aux grands centres de documentation et à des préoccupations de normalisation. Si les travaux dans le domaine des SOC commencent à s'ouvrir aux approches participatives, comme en témoigne l'intérêt pour les folksonomies (Ertzscheid et Gallezot, 2006; Hudon et El Hadi, 2010) et aux approches multipoints de vues et à facettes (Mas et Hudon, 2002 ; Marleau et al., 2009), la prise en compte de la pervasivité dans l'architecture de la connaissance n'en est qu'à ses balbutiements. A côté de la multiplication des travaux techniques dans le domaine des moteurs de recherche et des réseaux sémantiques (par exemple les ontologies), il manque des recherches qui intègrent la perspective des usages et les enjeux de pervasivité.

53 Ces recherches nécessitent simultanément la mise à disposition de cadres conceptuels renouvelés, auxquels nous espérons avoir contribué dans cet article, et des expérimentations basées sur la conception d'architectures innovantes et souvent 
hybrides, du type de celles développées dans le cadre du projet Miipa-Doc et brièvement présentées plus haut. Dans ces expérimentations, il ne s'agit pas d'accroître la performance absolue d'un composant isolé, mais de penser surtout l'intégration dans un environnement transmédia hétérogène permettant d'obtenir une organisation des connaissances pervasive en mesure de garantir la cohérence de l'information dans les actions opérationnelles comme dans les processus d'archivage.

\section{BIBLIOGRAPHIE}

Bachimont B., (2009), « Archivage audiovisuel et numérique : les enjeux de la longue durée », in C. Leblond (éd.), Archivage et stockage pérennes, Paris, Hermès, pp. 195-222.

Briet S., (1951), Qu'est-ce que la documentation?, Paris, Éditions documentaires, industrielles et techniques.

Chabin M.-A., (2010), Variations sur la version : un sujet de diplomatique numérique, Conférence-midi de l'EBSI, Montréal, $1^{\text {er }}$ novembre 2010, http://www.marieannechabin.fr/mac-et-sa-plume/ : (consulté le 01/05/2012).

Debray R., (2000), Introduction à la médiologie, Paris, Presses Universitaires de France.

Descola P., (2005), Par-delà nature et culture, Paris, Gallimard.

Desfriches Doria O., (2012), « Contribution de la classification à facette pour l'organisation des connaissances dans les organisations », Études de Communication, vol. 39 (à paraître).

Ertzscheid O. et Gallezot G., (2006), « Étude exploratoire des pratiques d'indexation sociale comme une renégociation des espaces documentaires. Vers un nouveau big bang documentaire ? ", in Chartron G. et Broudoux E. (éds.), Document numérique et société, Paris, ADBS Éditions.

Gnoli C. et Mazzocchi F. (éds.), (2010), Paradigms and conceptual systems in Knowledge Organization: Proceedings of the $11^{\text {th }}$ International Conference of the International Society for Knowledge Organization (ISKO), Rome, 23-26 Feb. 2010, Wurzburg, Ergon Verlag.

Hjørland B., (2008), « What is knowledge organization (KO)? », Knowledge Organization, vol. 35, n ०2/3, pp. 86-101.

Hoc J.-M., (1987), Psychologie cognitive de la planification, Grenoble, Presses Universitaires de Grenoble.

Hodge G., (2000), Systems of Knowledge Organization for Digital Libraries: Beyond Traditional Authority Files, The Digital Library Federation Council on Library and Information Resources, http:// www.clir.org/pubs/abstract/pub91abst.html.

Hudon M. et El Hadi W. M., (2010), « Organisation des connaissances et des ressources documentaires, De l'organisation hiérarchique centralisée à l'organisation sociale distribuée ", Les Cahiers du Numérique, vol. 6, $\mathrm{n}^{\circ}$ 3, pp. 9-38. 
Jenkins H., (2011), « Transmedia 202: Further Reflections », in Confessions of an Aca-Fan, The Official Weblog of Henry Jenkins, http://henryjenkins.org/2011/08/ defining_transmedia_further_re.html : (consulté le 02/05/2012).

Le Crosnier H. et Lecarpentier J.-M., (2010), Webdesign, normalisation \& stratégie des firmes, $13^{\mathrm{e}}$ colloque international sur le document électronique (CIDE 13), Document numérique, entre permanence et mutations, Paris, 16-17 décembre 2010.

Mas S. et Hudon M., (2002), « L'utilisation d'une classification à facettes pour l'organisation des documents institutionnels ", Congrès de l'ACFAS, Québec, 15 mai 2002.

Marleau Y., Mas S. et Zacklad M., (2009), « Exploitation des facettes et des ontologies sémiotiques pour la gestion documentaire », in Broudoux E. et Chartron G. (dir.), Actes de la deuxième conférence Document numérique et Société, Traitements et pratiques documentaires : vers un changement de paradigme ?, 17-18 novembre 2008, Paris, France, Paris, ADBS, pp. 91-110.

Meyriat J., (1981), Document, documentation, documentologie, Schéma et schématisation, $2^{\mathrm{e}}$ trimestre, $n^{\circ} 14$, pp. 51-63.

Pédauque R. T., (2006), Le document à la lumière du numérique, Caen, C\&F Éditions.

Zacklad M., (2004), « Processus de documentarisation dans les Documents pour l'Action (DopA) : statut des annotations et technologies de la coopération associées ", in Le numérique : Impact sur le cycle de vie du document pour une analyse interdisciplinaire, 13-15 Octobre 2004, Montréal, Québec, Villeurbanne, Éditions de l'ENSSIB.

Zacklad M., (2008), « Participative documentary spaces and governance », International Journal of Sustainable Development, vol. 11, n²/3/4, pp. 247-261.

Zacklad M., (2010a), « Sémiotique de la création de valeur dans l'économie des transactions coopératives ", in L'activité marchande sans le marché, Hatchuel A., Favereau O. et Aggeri F. (éds.), Paris, Presses de l'École des Mines, pp. 265-283.

Zacklad M., (2010b), «Évaluation des Systèmes d'Organisation des Connaissances », Les cahiers du numérique, vol. 6, $\mathrm{n}^{\circ}$ 3, pp. 133-166.

Zacklad M., Desfriches-Doria O., Bertin G., Mahe S., Ricard B., Musnik N., Cahier J.-P., Bénel A. et Lewkowicz E., (2011), « Miipa-Doc : gestion de l'hétérogénéité des classifications documentaires en entreprise ", in Hypermédias et pratiques numériques H2PTM'11, Paris, Hermes Science, pp. 223-243.

\section{RÉSUMÉS}

Dans cet article, nous défendons l'idée selon laquelle la généralisation des documents pour l'action numériques implique une réorientation des recherches dans le domaine de l'Organisation des Connaissances. Après un retour sur les notions de contenu et de support, nous introduisons la notion d'environnement-support d'un média et définissons la notion d'architecture des connaissances. Nous introduisons alors la notion de transmédia documentaire et ses déclinaisons, puis celle de pervasivité appliquée aux contenus et à l'Organisation des Connaissances qui les structure que nous illustrons à l'aide de résultats de recherche du projet ANR Miipa-Doc.

In this paper, we support the idea that the widespread use of digital documents for action implies a reorientation of research in the field of Knowledge Organization. We first look back on the concepts of content and substrate (support), before introducing the notions of substrate- 
environment (environnement-support) of a media and defining the notion of knowledge architecture. Then, we introduce the concept of transmedia document and its variations, as well as that of pervasiveness as applied to content and to the organization of knowledge that structures it. We illustrate these notions with results from the ANR Miipa-Doc research project.

INDEX

Mots-clés : organisation des connaissances, architecture des connaissances, transmédia documentaire, document pour l'action, pervasivité

Keywords : knowledge organization, knowledge architecture, transmedia document, document for action, pervasiveness

\section{AUTEUR}

\section{MANUEL ZACKLAD}

Conservatoire National des Arts et Métiers, Laboratoire DICEN EA 4420, CITS - 16DP30 - 292, rue Saint Martin 75141 Paris Cedex 03 